\section{Double stars}

Double Stars. By W. D. Heintz. Pp. 174. (D. Reidel: Dordrecht, The Netherlands, 1978.) Hardback Dfl.65; \$29; paperback Dfl.30; \$14.

DOUBLE STARS in the sky represent objects of great interest to the astronomer -not only because of their prodigious abundance (more than three-quarters of all stars around us seem to be multiple), but also because a symbiosis of their components provides the best data with which to document our theories on stellar evolution. Observations (positional photometric, spectroscopic) of binary systems have provided almost all we know on the masses and absolute dimensions of the stars, and, in addition, have enabled us to gain more subtle empirical insights into their structure.

In spite of these facts, the students of this important branch of astronomy have so far been served none too well by the existing contemporary literature. For this reason alone the appearance of the book under review is to be welcomed, because in many aspects it usefully fills a noticeable gap. However, this welcome cannot be extended to it without reservation concerning certain parts of its contents, where the latter falls short of the actual needs.

The present reviewer has nothing but praise for the first half of the book concerned with wide (visual or astrometric) binaries-a field in which its author is a recognised expert. That part of its text presents the first comprehensive picture of the field since $R$. G. Aitken's Binary Stars of 1935 (Dover reprint, 1964), brought up-to-date by an account of more recent developments (such as the use of electronic cameras, speckle interferometry, and so on). All this should be very useful to the student and researcher alike-not only by what it describes, but also by many references to the original sources which can guide its user in further reading.

With his account of the close (that is, spectroscopic and photometric) binaries, contained in the second half of the book, the author has been somewhat less fortunate-perhaps because its contents constitute largely a second-hand report which is in many places quite out of date. Thus, on the methodical side (determination of the elements) it does not go really beyond what one could find in Aitken's 1935 classic: Had there been no developments of the subject since that time, would it have been worthwhile to write another book about it? Of course they were; and some of them spectacular; but the author went past them without much notice.

Thus, the reader of Heintz's book will not learn much from it alone on the effects of distortion (or reflection) on the determination of masses or absolute dimensions of stars constituting close pairs. He will not learn that the process of "rectification" of their light curves represents an empirical make-shift, without theoretical justification, which is apt seriously to falsify the original light message. Hydrodynamical phenomena (gas streams) in close binary systems are still treated in terms of paricle mechanics (in which the pressure is ignored); and photometric effects are ascribed to them without appropriate inquiry as to the density of gas which could possess the requisite optical depth.

\section{Basic introduction to pesticides}

Pesticides: Preparation and Mode of Action. By R. Cremlyn, P. 240. (Wiley: Chichester, UK, and New York, 1978.) $£ 12$.

THIS book is aimed specifically at students who are taking first degrees or other qualifications in agriculture or related subjects. It covers, in separate chapters, insecticides, fungicides, herbicides, fumigants, rodenticides, nematicides, molluscicides, and the newer methods of insect control. The basic pattern of these chapters is to start with a brief history of how each particular group of pesticides came to be developed, to give brief details of synthetic routes, and to consider the biological activity.

As with so many agricultural subjects, a wide range of the traditional academic disciplines is involved, from organic chemistry to ecology. The book is obviously written by a chemist: I felt somewhat uneasy with some of the more biological statements. For example, "The nervous system is characteristic of mammals and insects ..." True, but incomplete, and the uninitiated might wonder which other groups of animals have nervous systems. I am not a professional chemist, but the accounts of synthetic routes seemed to be brief and competent. However, I was less happy with some other aspects of the chemistry. Thus, on p56 DDA is declared to be a metabolite of DDE-as far as I am aware DDA is metabolised only from DDT. Aldrin and dieldrin are both declared (on p64) to be chemically very stable: obviously it depends on how one defines stability, but dieldrin is normally regarded as more stable than aldrin.

These chapters are illustrated with
Moreover, in the last part of the book, the reader is being exposed to various evolutionary 'scenarios' without sufficient caution about their uncertainty or hypothetical nature. This part of the book is certainiy not a safe guide for the uninitiated. However, if it succeeds in making him at least interested enough in this field to follow it up by further reading (or work) of his own, it will have satisfied a very useful aim.

Zdenek Kopal

Zdenék Kopal is Professor of Astronomy in the University of Manchester, UK.

many structural formulae, which are useful, but identification is inconsistent. Some formulae are named, but many which can be identified by short names bear only a number. This number has to be discovered in the text before the identity of an unknown compound is revealed, which is tedious. There are also 28 black-and-white photographs, all taken by well-known manufacturers of pesticides, and mostly of crops with and without pesticide treatment. They are of somewhat marginal value.

Two introductory chapters discuss broader issues that apply to the whole range of pesticides. One, on physicochemical factors, discusses briefly the important topics of pesticide design, formulation and selectivity, application and behaviour of pesticides in the environment. The other covers biochemical reactions that are relevant to the mode of action of pesticides: respiration, photosynthesis, conduction of nerve impulses, transport in plants, synthesis of proteins.

The penultimate chapter considers pesticides in the environment, and crystallised some of the reactions I had experienced from the earlier chapters. Facts are often presented in a slightly misleading way, and often key references are absent. References are indeed rather few in number. They are placed at the end of each chapter, and the same standard texts such as Corbett, Hartley and West, Martin and O'Brien recur frequently. This book is of course more up-to-date than its predecessors, but does not replace them.

In summary, I cannot regard this as an authoritative book, but it may be useful for students who do not want to delve too deeply into any aspect of the subject.

F. Moriarty

F. Moriarty is a Principal Scientific Officer at the Institute of Terrestrial Ecology, Monks Wood Experimental Station, Huntingdon, UK. 